J. Appl. Numer. Optim. 2 (2020), No. 2, pp. 143-154

Available online at http://jano.biemdas.com

https://doi.org/10.23952/jano.2.2020.2.02

\title{
SOME NEW ITERATIVE ALGORITHMS FOR FINDING FIXED POINTS OF NONEXPANSIVE MAPPINGS
}

\author{
XIAO-HUAN LI ${ }^{1}$, QIAO-LI DONG ${ }^{1, *}$, AVIV GIBALI ${ }^{2,3}$ \\ ${ }^{1}$ Tianjin Key Lab for Advanced Signal Processing and College of Science, \\ Civil Aviation University of China, Tianjin 300300, China \\ ${ }^{2}$ Department of Mathematics, ORT Braude College, 2161002, Karmiel, Israel \\ ${ }^{3}$ The Center for Mathematics and Scientific Computation, \\ University of Haifa, Mt. Carmel, 3498838, Haifa, Israel
}

\begin{abstract}
In this paper, we introduce some new iterative algorithms for finding fixed points of nonexpansive mappings with the aid of projection and contraction methods. Weak and strong convergence theorems are established under mild conditions in Hilbert spaces. The numerical examples are presented to illustrate the advantage of our proposed algorithms.
\end{abstract}

Keywords. Nonexpansive mapping; Firmly nonexpansive mapping; Fixed point; Variational inequality.

\section{INTRODUCTION}

1.1. Projection methods for the variational inequality problem. The classical variational inequality (VI) problem is to find a point $x^{*} \in \mathscr{H}$ such that

$$
\left\langle F\left(x^{*}\right), x-x^{*}\right\rangle \geq 0, \text { for all } x \in C,
$$

where $C \subseteq \mathscr{H}$ is nonempty, closed and convex set in a real Hilbert space $\mathscr{H},\langle\cdot, \cdot\rangle$ denotes the inner product in $\mathscr{H}$, and $F: \mathscr{H} \rightarrow \mathscr{H}$ is a given mapping.

Assume that the following conditions hold:

Condition 1.1. The solution set of (1.1), denoted by $\operatorname{SOL}(C, F)$, is nonempty.

Condition 1.2. The mapping $F$ is monotone on $\mathscr{H}$, i.e.,

$$
\langle F(x)-F(y), x-y\rangle \geq 0, \quad \forall x, y \in \mathscr{H} .
$$

Condition 1.3. The mapping $F$ is Lipschitz continuous on $\mathscr{H}$ with the Lipschitz constant $L>0$, i.e.,

$$
\|F(x)-F(y)\| \leq L\|x-y\|, \quad \forall x, y \in \mathscr{H} .
$$

${ }^{*}$ Corresponding author.

E-mail addresses: xiaohuanlimath@163.com (X.H. Li), dongq1@1sec.cc.ac.cn (Q.L. Dong), avivg@braude.ac.il (A. Gibali).

Received July 26, 2019; Accepted October 8, 2019.

(C)2020 Journal of Applied and Numerical Optimization 
There are a great deal of projection methods for solving variational inequality (1.1), including well-known extragradient method proposed by Korpelevich [21]. Among these methods, projection and contraction methods [18] have excellent numerical behaviors and attracted much attention of researchers.

Algorithm 1.1 (Projection and contraction methods). Given $x^{k} \in \mathscr{H}$, compute

$$
y^{k}=P_{C}\left(x^{k}-\alpha_{k} F\left(x^{k}\right)\right),
$$

where $\alpha_{k} \in(0,1 / L)$ or $\alpha_{k}=\sigma \rho^{m_{k}}, \sigma>0, \rho \in(0,1)$ and $m_{k}$ is the smallest nonnegative integer such that

$$
\alpha_{k}\left\|F\left(x^{k}\right)-F\left(y^{k}\right)\right\| \leq \mu\left\|x^{k}-y^{k}\right\|, \quad \mu \in(0,1) .
$$

Let

$$
d\left(x^{k}, y^{k}\right)=\left(x^{k}-y^{k}\right)-\alpha_{k}\left(F\left(x^{k}\right)-F\left(y^{k}\right)\right),
$$

and

$$
\beta_{k}:=\frac{\varphi\left(x^{k}, y^{k}\right)}{\left\|d\left(x^{k}, y^{k}\right)\right\|^{2}}, \quad \varphi\left(x^{k}, y^{k}\right):=\left\langle x^{k}-y^{k}, d\left(x^{k}, y^{k}\right)\right\rangle,
$$

for each $k \geq 1$. Calculate

$$
x_{\mathrm{I}}^{k+1}=x^{k}-\gamma \beta_{k} d\left(x^{k}, y^{k}\right),
$$

or

where $\gamma \in(0,2)$.

$$
x_{\mathrm{II}}^{k+1}=P_{C}\left(x^{k}-\gamma \beta_{k} \alpha_{k} F\left(y^{k}\right)\right),
$$

In Algorithm 1.1, two choices for $x^{k+1}$ are given. There is one projection onto the $C$ if $x_{\mathrm{I}}^{k+1}$ is chosen and two projections onto the $C$ if $x_{\mathrm{II}}^{k+1}$ is chosen in the $(k+1)$-th iteration.

Dong, Cho and Rassias [12] extended Algorithm 1.1 to solve the common solutions of variational inequality problems. From [12, Theorem 3.1 and Theorem 3.2], it is easy to obtain the weak convergence of Algorithm 1.1 under the standard conditions.

Theorem 1.1. Let Conditions 1.1-1.3 hold. Then the sequence $\left\{x^{k}\right\}_{k=0}^{\infty}$ generated by Algorithm 1.1 converges weakly to a solution of variational inequality problem (1.1).

The computation of $x_{\mathrm{II}}^{k+1}$ involves the projection onto $C$, and, in general, the projection onto a convex set is difficult. Following the idea of Censor, Gibali and Reich [5], Dong, Jiang and Gibali [10] replaced $x_{\mathrm{II}}^{k+1}$ in (1.6) with

$$
x^{k+1}=P_{T_{k}}\left(x^{k}-\gamma \beta_{k} \alpha_{k} F\left(y^{k}\right)\right),
$$

where

$$
T_{k}:=\left\{w \in \mathscr{H} \mid\left\langle\left(x^{k}-\alpha_{k} F\left(x^{k}\right)\right)-y^{k}, w-y^{k}\right\rangle \leq 0\right\} .
$$

1.2. Krasnosel'skiǐ-Mann method for nonexpansive mappings. An operator $T: C \rightarrow C$ is said to be to be nonexpansive if

$$
\|T x-T y\| \leq\|x-y\|
$$

for all $x, y \in C$ and $\operatorname{Fix}(T):=\{x \in C: T x=x\}$ denotes the set of fixed points of $T$.

Next, we give the definition of the fixed point problem.

Problem 1.1. Suppose that $T: C \rightarrow C$ is a nonexpansive mapping with $\operatorname{Fix}(T) \neq \emptyset$.

Find a point $x^{*} \in C$ such that $T\left(x^{*}\right)=x^{*}$. 
Construction of common fixed points for a finite family of nonlinear mappings is of practical importance. In particular, iteration algorithms for finding common fixed points of a finite family of nonexpansive mappings have received vast investigation (see [1, 6, 28]) since these algorithms have a variety of application in inverse problem, image recovery, and signal processing (see [7, 27, 30, 31]).

The Krasnosel'skiǐ-Mann algorithm (KM) [25, 26] is one of the most used algorithms to solve the fixed point problem, which is defied as follows:

$$
x^{k+1}=\left(1-\lambda_{k}\right) x^{k}+\lambda_{k} T\left(x^{k}\right), \forall k \geq 0,
$$

where $T: \mathscr{H} \rightarrow \mathscr{H}$ is a nonexpansive operator in a Hilbert space $\mathscr{H}$ and $\lambda_{k} \in[0,1]$ for each $k \geq 1$. The iterative sequence $\left\{x^{k}\right\}$ converges weakly to a fixed point of a nonexpansive mapping $T$ provided that the relaxation parameter $\{\lambda\} \subset[0,1]$ satisfies the following condition:

$$
\sum_{k=1}^{\infty} \lambda_{k}\left(1-\lambda_{k}\right)=+\infty .
$$

Recently, Cruz [8] introduced the following algorithm to improve the convergence speed of KM iteration.

Algorithm 1.2. Step 0. Choose $x^{0} \in \mathscr{H}, 1 \ll \alpha_{\max }<\infty, \alpha_{0} \in\left(0, \alpha_{\max }\right], \gamma, \sigma \in(0,1)$ and a positive sequence $\left\{\eta_{k}\right\}$ such that

$$
\sum_{k=0}^{\infty} \eta_{k}=\eta<\infty
$$

Let $k:=0$ and $y^{0}=x^{0}-T x^{0}$.

Step 1. If $\left\|x^{k}-T x^{k}\right\|=0$, then stop.

Step 2. Compute $x^{k+1}, y^{k+1}$ and $\alpha_{k+1}$ as follows: Find $m_{k}$ as the smallest nonnegative integer number $m$ such that

$$
\left\|x^{k}-\sigma^{m} \alpha_{k} y^{k}-T\left(x^{k}-\sigma^{m} \alpha_{k} y^{k}\right)\right\|^{2} \leq\left(1-\gamma \sigma^{2 m}\right)\left\|y^{k}\right\|^{2}+\eta_{k} .
$$

Set $\lambda_{k}=\sigma^{m_{k}}, x^{k+1}=x^{k}-\lambda_{k} \alpha_{k} y^{k}, y^{k+1}=x^{k+1}-T x^{k+1}$ and

$$
\alpha_{n+1}= \begin{cases}\frac{\lambda_{k} \alpha_{k}\left\|y^{k}\right\|^{2}}{\left\|y^{k}\right\|^{2}-\left\langle y^{k+1}, y^{k}\right\rangle}, & \text { if }\left\langle y^{k+1}, y^{k}\right\rangle \leq\left(1-\frac{\alpha_{k}}{\alpha_{\max }}\right)\left\|y^{k}\right\|^{2}, \\ \alpha_{\max }, & \text { otherwise }\end{cases}
$$

Step 3. Set $k:=k+1$ and go to Step 1 .

To get the convergence of Algorithm 1.2, Cruz [8] assumed that $\Omega_{0}:=\left\{x \in \mathscr{H}:\|F x\|^{2} \leq\right.$ $\left.\left\|F x_{0}\right\|^{2}+\eta\right\}$ is compact, where $F: \mathscr{H} \rightarrow \mathscr{H}$ is defined as $F=I-T$. This assumption is strong and it is difficult to verify it.

KM iteration has only weak convergence (see [16] for an example). However, strong convergence is often much more desirable than the weak convergence in many problems that arise in infinite dimensional spaces (see [2] and references therein). So, attempts have been made to modify Krasnosel'skiǐ-Mann iteration algorithm so that strong convergence is guaranteed. To get the strong convergence of KM iterations, Nakajo and Takahashi [22] first introduced the following hybrid algorithm: 


\section{Algorithm 1.3.}

$$
\left\{\begin{array}{l}
x^{0} \in C \text { chosen arbitrarily, } \\
y^{k}=\alpha_{k} x^{k}+\left(1-\alpha_{k}\right) T x^{k} \\
C_{k}=\left\{z \in C:\left\|y^{k}-z\right\| \leq\left\|x^{k}-z\right\|\right\} \\
Q_{k}=\left\{z \in C:\left\langle x^{k}-z, x^{k}-x^{0}\right\rangle \leq 0\right\} \\
x^{k+1}=P_{C_{k} \cap Q_{k}} x^{0}
\end{array}\right.
$$

The Algorithm 1.3 strongly converges to $P_{\mathrm{Fix}(\mathrm{T})} x^{0}$. There are some modifications and improvement for the hybrid method 1.3 (see, e.g. [9, 11]).

Recently, some authors [4, 13, 15] investigated the inertial KM algorithms by combining KM iterations and inertial techniques. Very recently, Dong et al. [14] firstly introduced the multi-step inertial KM iterations (MiKM) and showed the numerical advantage of the MiKM.

\section{PRELIMinaries}

We use the following notations:

(i) $\rightarrow$ for weak convergence and $\rightarrow$ for strong convergence;

(ii) $\omega_{w}\left(x^{k}\right)=\left\{x: \exists x^{k_{j}} \rightarrow x\right\}$ denotes the weak $\omega$-limit set of $\left\{x^{k}\right\}$, that is, any $x \in \omega_{w}\left(x^{k}\right)$ such that there exists a subsequence $\left\{x^{k_{j}}\right\}_{j=0}^{\infty}$ of $\left\{x^{k}\right\}_{k=0}^{\infty}$ which converges weakly to $x$.

Let $C$ be a nonempty, closed and convex subset of $\mathscr{H}$. For each point $x \in \mathscr{H}$, there exists a unique nearest point in $C$, denoted by $P_{C}(x)$, such that

$$
\left\|x-P_{C}(x)\right\| \leq\|x-y\| \text { for all } y \in C .
$$

The mapping $P_{C}: \mathscr{H} \rightarrow C$ is called the metric projection of $\mathscr{H}$ onto $C$. It is known that $P_{C}$ is a nonexpansive mapping of $\mathscr{H}$ onto $C$, and further more firmly nonexpansive mapping. This is captured in the next lemma.

Lemma 2.1. For any $x, y \in \mathscr{H}$ and $z \in C$, it holds

- $\left\|P_{C}(x)-P_{C}(y)\right\| \leq\|x-y\|$;

- $\left\|P_{C}(x)-z\right\|^{2} \leq\|x-z\|^{2}-\left\|P_{C}(x)-x\right\|^{2}$;

The characterization of the metric projection $P_{C}[17$, Section 3] is given in the next lemma.

Lemma 2.2. Let $x \in \mathscr{H}$ and $z \in C$. Then $z=P_{C}(x)$ if and only if

$$
\left\langle x-P_{C}(x), P_{C}(x)-y\right\rangle \geq 0 \text { for all } x \in \mathscr{H}, y \in C .
$$

Give $x \in \mathscr{H}$ and $v \in \mathscr{H}, v \neq 0$ and let $T=\{z \in \mathscr{H}:\langle v, z-x\rangle \leq 0\}$. Then, for all $u \in \mathscr{H}$, the projection $P_{T}(u)$ is defined by

$$
P_{T}(u)=u-\max \left\{0, \frac{\langle v, u-x\rangle}{\|v\|^{2}}\right\} v,
$$

which gives us an explicit formula to find the projection of any point onto a half-space (see [19] for details). 
Definition 2.1. The normal cone of $C$ at $v \in C$, denote by $N_{C}(v)$ is defined as

$$
N_{C}(v):=\{d \in \mathscr{H} \mid\langle d, y-v\rangle \leq 0 \text { for all } y \in C\} .
$$

Definition 2.2. Let $B: \mathscr{H} \rightrightarrows 2^{\mathscr{H}}$ be a point-to-set operator defined on a real Hilbert space $\mathscr{H}$. The operator $B$ is called a maximal monotone operator if $B$ is monotone, i.e.,

$$
\langle u-v, x-y\rangle \geq 0 \text { for all } u \in B(x) \text { and } v \in B(y),
$$

and the graph $G(B)$ of $B$,

$$
G(B):=\{(x, u) \in \mathscr{H} \times \mathscr{H} \mid u \in B(x)\},
$$

is not properly contained in the graph of any other monotone operator.

It is clear ([23, Theorem 3]) that a monotone mapping $B$ is maximal if and only if, for any $(x, u) \in \mathscr{H} \times \mathscr{H}$, if $\langle u-v, x-y\rangle \geq 0$ for all $(v, y) \in G(B)$, then it follows that $u \in B(x)$.

Lemma 2.3. [3] Let $K$ be a nonempty, closed and convex subset of a Hilbert space $\mathscr{H}$. Let $\left\{x^{k}\right\}_{k=0}^{\infty}$ be a bounded sequence which satisfies the following properties:

- every limit point of $\left\{x^{k}\right\}_{k=0}^{\infty}$ lies in $K$;

- $\lim _{n \rightarrow \infty}\left\|x^{k}-x\right\|$ exists for every $x \in K$.

Then $\left\{x^{k}\right\}_{k=0}^{\infty}$ weakly converges to a point in $K$.

\section{AlgORITHMS AND THE CONVERGENCE ANALYSIS}

In this section, we give some iterative algorithms based on the projection and contraction algorithms of the variational inequalities.

\subsection{Some iterative methods with weak convergence. Let}

$$
F:=I-T \text {. }
$$

Lemma 3.1. Let $T: \mathscr{H} \rightarrow \mathscr{H}$ be a nonexpansive mapping. Then $F$ is monotone and 2-Lipschitz continuous.

Proof. For $\forall x, y \in \mathscr{H}$, from the nonexpansivity of $T$, we have

$$
\begin{aligned}
\langle F(x)-F(y), x-y\rangle & =\|x-y\|^{2}-\langle T(x)-T(y), x-y\rangle \\
& \geq\|x-y\|(\|x-y\|-\|T(x)-T(y)\|) \geq 0,
\end{aligned}
$$

which implies that $F$ is monotone.

Using the nonexpansivity of $T$ again, we have

$$
\begin{aligned}
\|F(x)-F(y)\| & =\|(x-y)-(T(x)-T(y))\| \\
& \leq\|x-y\|+\|T(x)-T(y)\| \\
& \leq 2\|x-y\| .
\end{aligned}
$$

So, $F$ is 2-Lipschitz continuous.

Lemma 3.2. Let $T: C \rightarrow C$ be a nonexpansive mapping with $\operatorname{Fix}(T) \neq \emptyset$. Then $\operatorname{Fix}(T)=$ $\operatorname{SOL}(C, F)$. 
Proof. It is easy to verify that $\operatorname{Fix}(T) \subseteq S O L(C, F)$. Next we show $\operatorname{SOL}(C, F) \subseteq$ Fix $(T)$. Take arbitrarily $x^{*} \in S O L(C, F) \subseteq C$. For $\forall \alpha \in(0,1)$, from (1.1), it follows

$$
\left\langle x^{*}-\left(x^{*}-\alpha F\left(x^{*}\right)\right), x-x^{*}\right\rangle \geq 0, \text { for all } x \in C,
$$

Using Lemma 2.2, we have

$$
x^{*}=P_{C}\left(x^{*}-\alpha F\left(x^{*}\right)\right) \text {. }
$$

By the convexity of $C$ and $\alpha \in(0,1)$, we have

$$
x^{*}-\alpha F\left(x^{*}\right)=(1-\alpha) x^{*}+\alpha T\left(x^{*}\right) \in C .
$$

Therefore, (3.1) yields $x^{*}=x^{*}-\alpha F\left(x^{*}\right)$, i.e., $F\left(x^{*}\right)=0$. We get $x^{*}=T\left(x^{*}\right)$. Hence $x^{*} \in$ $\operatorname{Fix}(T)$. So, $\operatorname{SOL}(C, F) \subseteq \operatorname{Fix}(T)$.

Algorithm 3.1. Take arbitrarily $x^{0} \in C$ and calculate

$$
\left\{\begin{array}{l}
y^{k}=\left(1-\alpha_{k}\right) x^{k}+\alpha_{k} T\left(x^{k}\right), \\
x^{k+1}=P_{C}\left(x^{k}-\gamma \rho_{k} d\left(x^{k}, y^{k}\right)\right),
\end{array}\right.
$$

where $\gamma \in(0,2)$, and

$$
\rho_{k}:=\frac{\left\langle x^{k}-y^{k}, d\left(x^{k}, y^{k}\right)\right\rangle}{\left\|d\left(x^{k}, y^{k}\right)\right\|^{2}}
$$

where

$$
d\left(x^{k}, y^{k}\right)=\left(x^{k}-y^{k}\right)-\alpha_{k}\left(F\left(x^{k}\right)-F\left(y^{k}\right)\right),
$$

the stepsize $\alpha_{k}$ is chosen as in Algorithm 1.1, where $\sigma \in(0,1)$.

The inner loop of the stepsize calculation in (1.4) is always finite and that the denominator in the definition of $\alpha_{k}$ is nonzero.

Lemma 3.3. [20] The line rule (1.4) is well defined. Besides, $\underline{\alpha} \leq \alpha_{k} \leq \sigma$, where $\underline{\alpha}=$ $\min \left\{\sigma, \frac{\mu \rho}{2}\right\}$.

Theorem 3.1. Let $T: C \rightarrow C$ be a nonexpansive mapping with $\operatorname{Fix}(T) \neq \emptyset$. Assume that $\sigma \in$ $(0,1)$. Then the sequence $\left\{x^{k}\right\}_{k=0}^{\infty}$ generated by Algorithm 3.1 converges weakly to a point in $\operatorname{Fix}(T)$.

Proof. Using Lemma 3.3 and $\sigma \in(0,1)$, we get $\alpha_{k} \leq \sigma<1$. From (3.2), we have

$$
x^{k}-\alpha_{k} F\left(x^{k}\right)=\left(1-\alpha_{k}\right) x^{k}+\alpha_{k} T\left(x^{k}\right) \in C .
$$

Using Lemmas 3.1 and 3.2, and Theorem 1.1, the proof is completed.

Algorithm 3.2. Take arbitrarily $x^{0} \in C$ and calculate

$$
\left\{\begin{array}{l}
y^{k}=\left(1-\alpha_{k}\right) x^{k}+\alpha_{k} T\left(x^{k}\right), \\
x^{k+1}=P_{C}\left(x^{k}-\gamma \rho_{k} \alpha_{k} F\left(y^{k}\right)\right),
\end{array}\right.
$$

where $\gamma \in(0,2)$, the stepsize $\alpha_{k}$ chosen as in Algorithm 1.1, and

$$
\rho_{k}:=\frac{\left\langle x^{k}-y^{k}, d\left(x^{k}, y^{k}\right)\right\rangle}{\left\|d\left(x^{k}, y^{k}\right)\right\|^{2}},
$$

where

$$
d\left(x^{k}, y^{k}\right)=\left(x^{k}-y^{k}\right)-\alpha_{k}\left(F\left(x^{k}\right)-F\left(y^{k}\right)\right) .
$$


By using Theorem 1.1, we easily show the weak convergence of Algorithm 3.2.

Theorem 3.2. Let $T: C \rightarrow C$ be a nonexpansive mapping with $\operatorname{Fix}(T) \neq \emptyset$. Assume that $\sigma \in$ $(0,1)$. Then the sequence $\left\{x^{k}\right\}_{k=0}^{\infty}$ generated by Algorithm 3.2 converges weakly to a point in $\operatorname{Fix}(T)$.

Remark 3.1. Algorithm 3.1 and Algorithm 3.2 involve a projection onto set $C$ which may lead to difficulty in the actual calculation. One way to overcome is to replace the projection onto set $C$ with a projection onto a halfspace [10].

Next, we introduce two new method which does not involve the projection onto set $C$.

Algorithm 3.3. Take arbitrarily $x^{0} \in \mathscr{H}$ and calculate

$$
\left\{\begin{array}{l}
y^{k}=\left(1-\alpha_{k}\right) x^{k}+\alpha_{k} T\left(x^{k}\right), \\
x^{k+1}=x^{k}-\gamma \rho_{k} d\left(x^{k}, y^{k}\right),
\end{array}\right.
$$

where $\gamma \in(0,2)$, the stepsize $\alpha_{k}$ chosen as in Algorithm 1.1, and

$$
\rho_{k}:=\frac{\left\langle x^{k}-y^{k}, d\left(x^{k}, y^{k}\right)\right\rangle}{\left\|d\left(x^{k}, y^{k}\right)\right\|^{2}}
$$

where

$$
d\left(x^{k}, y^{k}\right)=\left(x^{k}-y^{k}\right)-\alpha_{k}\left(F\left(x^{k}\right)-F\left(y^{k}\right)\right) .
$$

Theorem 3.3. Let $T: \mathscr{H} \rightarrow \mathscr{H}$ be a nonexpansive mapping with $\operatorname{Fix}(T) \neq \emptyset$. Assume that $\sigma \in(0,1)$. Then the sequence $\left\{x^{k}\right\}_{k=0}^{\infty}$ generated by Algorithm 3.3 converges weakly to a point in $\operatorname{Fix}(T)$.

Algorithm 3.4. Take arbitrarily $x^{0} \in \mathscr{H}$ and calculate

$$
\left\{\begin{array}{l}
y^{k}=\left(1-\alpha_{k}\right) x^{k}+\alpha_{k} T\left(x^{k}\right) \\
x^{k+1}=x^{k}-\gamma \rho_{k} \alpha_{k} y^{k}+\gamma \rho_{k} \alpha_{k} T\left(y^{k}\right)
\end{array}\right.
$$

where $\gamma \in(0,2)$, the stepsize $\alpha_{k}$ chosen as in Algorithm 1.1, and

$$
\rho_{k}:=\frac{\left\langle x^{k}-y^{k}, d\left(x^{k}, y^{k}\right)\right\rangle}{\left\|d\left(x^{k}, y^{k}\right)\right\|^{2}},
$$

where

$$
d\left(x^{k}, y^{k}\right)=\left(1-\alpha_{k}\right)\left(x^{k}-y^{k}\right)+\alpha_{k}\left(T\left(x^{k}\right)-T\left(y^{k}\right)\right) .
$$

Theorem 3.4. Let $T: \mathscr{H} \rightarrow \mathscr{H}$ be a nonexpansive mapping with $\operatorname{Fix}(T) \neq \emptyset$. Assume that $\sigma \in(0,1)$. Then the sequence $\left\{x^{k}\right\}_{k=0}^{\infty}$ generated by Algorithm 3.4 converges weakly to a point in $\operatorname{Fix}(T)$.

3.2. Some hybrid methods. By combining the hybrid technique, we introduce some iterative algorithms with strong convergence. 
Algorithm 3.5. Take arbitrarily $x^{0} \in C$ and calculate

$$
\left\{\begin{array}{l}
y^{k}=\left(1-\alpha_{k}\right) x^{k}+\alpha_{k} T\left(x^{k}\right), \\
w^{k}=P_{C}\left(x^{k}-\gamma \rho_{k} d\left(x^{k}, y^{k}\right)\right) \\
C_{k}=\left\{v \in C:\left\|w^{k}-v\right\|^{2} \leq\left\|x^{k}-v\right\|^{2}-\gamma(2-\gamma) \rho_{k} \varphi_{k}\right\} \\
Q_{k}=\left\{v \in C:\left\langle x^{0}-x^{k}, v-x^{k}\right\rangle \leq 0\right\} \\
x^{k+1}=P_{C_{k} \cap Q_{k}} x^{0}
\end{array}\right.
$$

where $\gamma, \rho_{k}, d\left(x^{k}, y^{k}\right)$ and $\alpha_{k}$ given as in Algorithm 3.1, and

$$
\varphi_{k}=\left\langle x^{k}-y^{k}, d\left(x^{k}, y^{k}\right)\right\rangle .
$$

Employing the proof of [12, Theorem 4.1], we get the following convergent result of Algorithm 3.5 .

Theorem 3.5. Let $T: C \rightarrow C$ be a nonexpansive mapping with $\operatorname{Fix}(T) \neq \emptyset$. Assume that $\sigma \in$ $(0,1)$. Then the sequence $\left\{x^{k}\right\}_{k=0}^{\infty}$ generated by Algorithm 3.5 converges strongly to $P_{\mathrm{Fix}(\mathrm{T})} x^{0}$.

Algorithm 3.6. Take arbitrarily $x^{0} \in C$ and calculate

$$
\left\{\begin{array}{l}
y^{k}=\left(1-\alpha_{k}\right) x^{k}+\alpha_{k} T\left(x^{k}\right), \\
w^{k}=P_{C}\left(x^{k}-\gamma \rho_{k} \alpha_{k} F\left(y^{k}\right)\right), \\
C_{k}=\left\{v \in C:\left\|w^{k}-v\right\|^{2} \leq\left\|x^{k}-v\right\|^{2}-\gamma(2-\gamma) \rho_{k} \varphi_{k}\right\} \\
Q_{k}=\left\{v \in C:\left\langle x^{0}-x^{k}, v-x^{k}\right\rangle \leq 0\right\} \\
x^{k+1}=P_{C_{k} \cap Q_{k}} x^{0}
\end{array}\right.
$$

where $\gamma, \rho_{k}, d\left(x^{k}, y^{k}\right)$ and $\alpha_{k}$ given as in Algorithm 3.2, and $\varphi_{k}$ defined as in (3.16).

Theorem 3.6. Let $T: C \rightarrow C$ be a nonexpansive mapping with $\operatorname{Fix}(T) \neq \emptyset$. Assume that $\sigma \in$ $(0,1)$. Then the sequence $\left\{x^{k}\right\}_{k=0}^{\infty}$ generated by Algorithm 3.6 converges strongly to $P_{\mathrm{Fix}(\mathrm{T})} x^{0}$.

Algorithm 3.7. Take arbitrarily $x^{0} \in \mathscr{H}$ and calculate

$$
\left\{\begin{array}{l}
y^{k}=\left(1-\alpha_{k}\right) x^{k}+\alpha_{k} T\left(x^{k}\right) \\
w^{k}=x^{k}-\gamma \rho_{k} d\left(x^{k}, y^{k}\right) \\
C_{k}=\left\{v \in \mathscr{H}:\left\|w^{k}-v\right\|^{2} \leq\left\|x^{k}-v\right\|^{2}-\gamma(2-\gamma) \rho_{k} \varphi_{k}\right\} \\
Q_{k}=\left\{v \in \mathscr{H}:\left\langle x^{0}-x^{k}, v-x^{k}\right\rangle \leq 0\right\} \\
x^{k+1}=P_{C_{k} \cap Q_{k}} x^{0}
\end{array}\right.
$$

where $\gamma, \rho_{k}, d\left(x^{k}, y^{k}\right)$ and $\alpha_{k}$ given as in Algorithm 3.3, and $\varphi_{k}$ defined as in (3.16).

Theorem 3.7. Let $T: \mathscr{H} \rightarrow \mathscr{H}$ be a nonexpansive mapping with $\operatorname{Fix}(T) \neq \emptyset$. Assume that $\sigma \in$ $(0,1)$. Then the sequence $\left\{x^{k}\right\}_{k=0}^{\infty}$ generated by Algorithm 3.7 converges strongly to $P_{\mathrm{Fix}(\mathrm{T})} x^{0}$. 
Algorithm 3.8. Take arbitrarily $x^{0} \in \mathscr{H}$ and calculate

$$
\left\{\begin{array}{l}
y^{k}=\left(1-\alpha_{k}\right) x^{k}+\alpha_{k} T\left(x^{k}\right) \\
w^{k}=x^{k}-\gamma \rho_{k} \alpha_{k} y^{k}+\gamma \rho_{k} \alpha_{k} T\left(y^{k}\right) \\
C_{k}=\left\{v \in \mathscr{H}:\left\|w^{k}-v\right\|^{2} \leq\left\|x^{k}-v\right\|^{2}-\gamma(2-\gamma) \rho_{k} \varphi_{k}\right\} \\
Q_{k}=\left\{v \in \mathscr{H}:\left\langle x^{0}-x^{k}, v-x^{k}\right\rangle \leq 0\right\} \\
x^{k+1}=P_{C_{k} \cap Q_{k}} x^{0}
\end{array}\right.
$$

where $\gamma, \rho_{k}, d\left(x^{k}, y^{k}\right)$ and $\alpha_{k}$ given as in Algorithm 3.4, and $\varphi_{k}$ defined as in (3.16).

Theorem 3.8. Let $T: \mathscr{H} \rightarrow \mathscr{H}$ be a nonexpansive mapping with $\operatorname{Fix}(T) \neq \emptyset$. Assume that $\sigma \in$ $(0,1)$. Then the sequence $\left\{x^{k}\right\}_{k=0}^{\infty}$ generated by Algorithm 3.8 converges strongly to $P_{\mathrm{Fix}(\mathrm{T})} x^{0}$.

\section{NUMERICAL EXAMPLES}

In this section, we present a numerical example to illustrate the behaviors of Algorithm 3.4 and Krasnosel'skiî-Mann iteration. All the programs are written in Matlab version 7.0. and performed on a PC Desktop Intel(R) Core(TM) i5-4200U CPU @ 1.60GHz 2.30 GHz, RAM 4.00 GB.

Problem 4.1. [24] For any nonempty closed convex set $K_{i} \subset \mathbb{R}^{N}$ for each $i=0,1, \cdots, m$,

$$
\text { find } x^{*} \in K:=\bigcap_{i=0}^{m} K_{i},
$$

where one assumes that $K \neq \emptyset$.

Define a mapping $T: \mathbb{R}^{N} \rightarrow \mathbb{R}^{N}$ by

$$
T:=P_{0}\left(\frac{1}{m} \sum_{i=1}^{m} P_{i}\right)
$$

where $P_{i}=P_{K_{i}}(i=0,1, \cdots, m)$ stands for the metric projection onto $K_{i}$. Since $P_{i}(i=0,1, \cdots, m)$ is nonexpansive, the mapping $T$ defined by (4.1) is also nonexpansive. Moreover, we find that

\begin{tabular}{|c|c|c|c|c|}
\hline \multirow[b]{2}{*}{$(N, m)$} & \multicolumn{2}{|c|}{ Algorithm 3.3(3.4) } & \multicolumn{2}{|c|}{ KM iteration } \\
\hline & Iter. & Sec. & Iter. & Sec. \\
\hline$(100,50)$ & 38 & 0.079298 & 378 & 0.08 \\
\hline$(50,100)$ & 77 & 0.22849 & 800 & 0.26983 \\
\hline$(100,100)$ & 70 & 0.22219 & 697 & 0.2803 \\
\hline$(100,500)$ & 264 & 2.7284 & 2368 & 3.9372 \\
\hline$(500,100)$ & 64 & 0.52328 & 588 & 0.6021 \\
\hline
\end{tabular}

$$
\operatorname{Fix}(T)=F i x\left(P_{0}\right) \bigcap_{i=1}^{m} \operatorname{Fix}\left(P_{i}\right)=K_{0} \bigcap_{i=1}^{m} K_{i}=K \text {. }
$$

TABLE 1. Comparison of Algorithm 3.4 and KM iteration. 


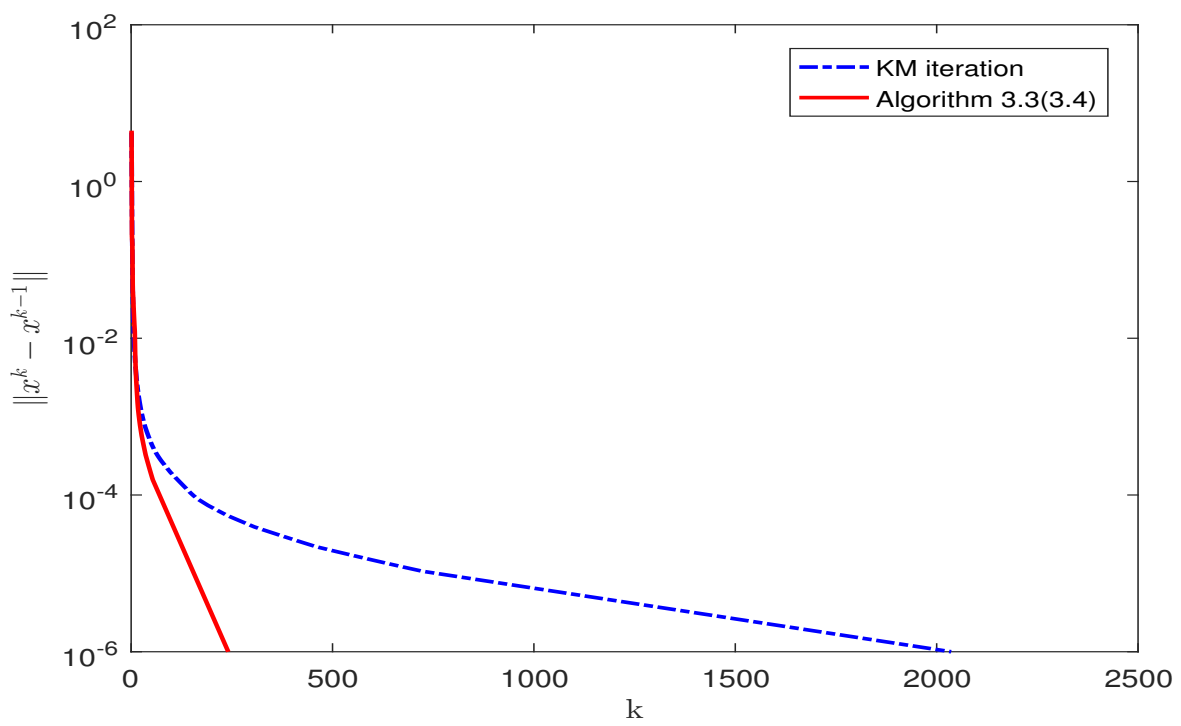

FIGURE 1. Comparison of Algorithm 3.4 and Mann Iteration.

In the experiment, we set $K_{i}(i=0,1, \cdots, m)$ as a closed ball with center $c_{i} \in \mathbb{R}^{N}$ and radius $r_{i}>0$. Thus $P_{i}(i=0,1, \cdots, m)$ can be computed with

$$
P_{i}(x):= \begin{cases}c_{i}+\frac{r_{i}}{\left\|c_{i}-x\right\|}\left(x-c_{i}\right) & \text { if } \quad\left\|c_{i}-x\right\|>r_{i}, \\ x & \text { if }\left\|c_{i}-x\right\| \leq r_{i} .\end{cases}
$$

We take $r_{i}:=1(i=0,1, \cdots, m), c_{0}:=0$. And $c_{i} \in(-1 / \sqrt{N}, 1 / \sqrt{N})^{N}(i=1, \cdots, m)$ are randomly chosen.

In the numerical results listed in the following tables, "Iter." denotes the number of iterations and "Sec." denotes the CPU time. We take $\left\|x^{k}-x^{k-1}\right\|<10^{-6}$ as the stopping criterion and choose $\gamma=1.9, \mu=0.5, \sigma=7$ and $\rho=0.6$ in Algorithm 3.4 and $\alpha_{k}=0.9$ in Mann Iteration. Take $m=100, N=500$ in Figure 1 .

As we see from Table 1 and Figure 1 the behaviors of Algorithm 3.3 and Algorithm 3.4 are the same, and they are better than KM iteration from the number of iterations and the iteration time.

\section{CONCLUSION}

In this paper, by using the equivalence of the nonexpansive operator and monotone and Lipschitz continuous operator, we gave eight iterative algorithms for fixed points of nonexpansive mappings. The numerical example illustrates the advantage of our proposed algorithms. There are other methods for the variational inequalities, such as, Tseng's forward-backward-forward method, which has good numerical performance [29]. It is thus natural to consider these method to approximate fixed points of nonexpansive mappings or related operators.

\section{Acknowledgements}


The authors of this paper are thankful to the referees for their constructive comments. The second author was supported by Fundamental Research Funds for the Central Universities (No. 201919).

\section{REFERENCES}

[1] S. Atsushiba, W. Takahashi, Strong convergence theorems for a fiite family of nonexpansive mappings and applications, Indian J. Math. 41 (1999), 435-453.

[2] H.H. Bauschke, The approximation of fixed points of compositions of nonexpansive mappings in Banach spaces, J. Math. Anal. Appl. 202 (1996), 150-159.

[3] H.H. Bauschke, P.L. Combettes, Convex Analysis and Monotone Operator Theory in Hilbert Spaces, Springer, Berlin, 2011.

[4] R.I. Bot, E.R. Csetnek, C. Hendrich, Inertial Douglas-Rachford splitting for monotone inclusion problems, Appl. Math. Comput. 256 (2015), 472-487.

[5] Y. Censor, A. Gibali, S. Reich, The subgradient extragradient method for solving variational inequalities in Hilbert space, J. Optim. Theory Appl. 148 (2011), 318-335.

[6] Y.J. Cho, S.M. Kang, X. Qin, Approximation of common fixed points of an infinite family of nonexpansive mappings in Banach spaces, Comput. Math. Appl. 56 (2008), 2058-2064.

[7] P.L. Combettes, On the numerical robustness of the parallel projection method in signal synthesis, IEEE Signal Proc. Let. 8 (2001), 45-47.

[8] W.L. Cruz, A residual algorithm for finding a fixed point of a nonexpansive mapping, J. Fixed Point Theory Appl. 20 (2018), 116.

[9] Q.L. Dong, S. He, Y.J. Cho, A new hybrid algorithm and its numerical realization for two nonexpansive mappings, Fixed Point Theory Appl. 1 (2015), 150.

[10] Q.L. Dong, D. Jiang, A. Gibali, A modified subgradient extragradient method for solving the variational inequality, Numerical Algor. 79 (2018), 927-940.

[11] Q.L. Dong, Y.Y. Lu, A new hybrid algorithm for a nonexpansive mapping, Fixed Point Theory Appl. 2015 (2015), 7.

[12] Q.L. Dong, Y. Cho, TH.M. Rassias, The projection and contraction methods for finding common solutions to variational inequality problems, Optim. Lett. 12 (2018), 1871-1896.

[13] Q.L. Dong, Y.J. Cho, Th.M. Rassias, General inertial Mann algorithms and their convergence analysis for nonexpansive mappings, In: T.M. Rassias, (ed.) Applications of Nonlinear Analysis, pp. 175-191, Springer, 2018.

[14] Q.L. Dong, J. Huang, X.H. Li, Y.J. Cho, Th.M. Rassias, MiKM: Multi-step inertial Krasnosel'skiǐ-Mann algorithm and its applications, J. Global Optim. 73 (2019), 801-824.

[15] Q.L. Dong, H.B. Yuan, Y.J. Cho, Th.M. Rassias, Modified inertial Mann algorithm and inertial CQ-algorithm for nonexpansive mappings, Optim. Lett. 12 (2018), 87-102.

[16] A. Genel, J. Lindenstrass, An example concerning fixed points, Isreal J. Math. 22 (1975), 81-86.

[17] K. Goebel, S. Reich, Uniform Convexity, Hyperbolic Geometry, and Nonexpansive Mappings, Marcel Dekker, New York, 1984.

[18] B.S., He, B.S, A class of projection and contraction methods for monotone variational inequalities, Appl. Math. Optim. 35 (1997), 69-76.

[19] S. He, C. Yang, P. Duan, Realization of the hybrid method for Mann iterations, Appl. Math. Comput. 217 (2010), 4239-4247.

[20] E.N. Khobotov, Modification of the extragradient method for solving variational inequalities and certain optimization problems, USSR Comput. Math. Math. Phys. 27 (1987), 120-127.

[21] G.M. Korpelevich, The extragradient method for finding saddle points and other problems, Ekon. Mat. Metody 12(1976), 747-756.

[22] K. Nakajo, W. Takahashi, Strong convergence theorems for nonexpansive mappings and nonexpansive semigroups, J. Math. Anal. Appl. 279 (2003), 372-379.

[23] R.T. Rockafellar, Monotone operators and the proximal point algorithm, SIAM J. Control Optim. 14 (1976), 877-898. 
[24] K. Sakurai, H. Liduka, Acceleration of the Halpern algorithm to search for a fixed poin of a nonexpansive mapping, Fixed Point Theory Appl. 2014 (2014), 202.

[25] W.R. Mann, Mean value methods in iteration, Proc. Amer. Math. Soc. 4 (1953), 506-510.

[26] M.A. Krasnosel'skiǐ, Two remarks on the method of successive approximations, Usp. Mat. Nauk 10 (1955), 123-127.

[27] C.I. Podilchuk, R.J. Mammone, Image recovery by convex projections using a least-squares constraint, J. Optim. Soc. Amer. 7 (1990), 517-512.

[28] X. Qin, J.C. Yao, Projection splitting algorithms for nonself operators, J. Nonlinear Convex Anal. 18 (2017), 925-935.

[29] P. Tseng. A modified forward-backward splitting method for maximal monotone mappings. SIAM J. Control Optim. 38(2) (2018), 431-446

[30] H.K. Xu, A variable Krasnosel'skii-Mann algorithm and the multiple-set split feasibility problem, Inverse Probl. 22 (2006), 2021-2034.

[31] D. Youla, Mathematical theory of image restoration by the method of convex projection, In: H. Stark, (ed.) Image Recovery Theory and Applications, pp. 29-77, Academic Press, Orlando, 1987. 